\title{
Confection of an occlusal stability intra-oral device for violin players
}

\author{
Agostinho Martins dos Santos \\ Faculty of dental medicine university of oporto, Portugal
}

\begin{abstract}
Objectives: Idealization and confection of an intra-oral device capable of providing occlusal stability and muscle tension relief referred by the violin players.

Methods: Dental impression of both teeth arches, face bow register as well as a register of the intermaxillary relation, in their most usual playing position, were made from the violinists for assemble of the teeth casts in the Protar 7 articulator. The individualized intra-oral device was made with the Erko-Loc Pro 3mm plates. The device was adjusted in the articulator at first and then intra orally with the T-scan device during violin playing.

Results: We achieved a device capable of avoiding teeth grinding, muscular tension relief, during violin playing, and a sensation of stability by having all teeth contacting.

Conclusion: We concluded that the usage of an intra-oral device might prove to be effective in reducing muscle hypertonia and preventing temporomandibular disorders, due to the improvements in occlusal stability given with same intensity contacts throughout all the teeth as well as a relief in symptoms of orofacial muscle pain. To clarify this concepts there is a need for more studies.

Clinical relevance: The study of the mandibular cinematic has implications on different areas of dental medicine. Movement patterns depend on the muscles, temporomandibular joint and associated structures.

Violin players rotate the mandible to the side in which they rest the violin to better stabilize it with the shoulder. This lateral position can be harmful to the masticatory muscles and to the temporomandibular joint.
\end{abstract}

\section{Background}

The study of the mandibular cinematic has important applications and its fundamental in different areas of dental medicine, due to its pervasiveness, giving not only the scientific basis of oral rehabilitation, but also the stability of the stomatognathic system. Movement patterns depend on the muscles, TMJ and associated structures [1-4].

Throughout the centuries violinists changed their playing position. The initial positions were of experimentation and had much to do with etiquette and appearances. Then, as the technique demanded, gradually they shifted to position that allowed more complexity and at the same time to play more comfortably for longer time periods. Important to say that the instruments were modified as well gradually according to these requirements and that reflects on the way of playing and holding the instrument [5].

Modern violinists, while holding the violin, rotate the mandible to side in which they rest the violin to better stabilize with the shoulder. This lateral position can be harmful to the masticatory muscles and to the temporomandibular joint, due to the long periods of time that it is maintained, with an eccentric isometric contraction that can be the origin of a muscular discoordination with an alteration of the muscle bioelectrical potentials (MBP) and instability both occlusal and articular [6] (Figure 1).

Nowadays, the great majority of violinists complaints of developing too much muscle tension, primarily in the shoulder that supports the violin e the masseter, medial pterygoid and temporal muscles due to the isometric tension exercised and even referring teeth grinding during violin practice [6].

It was intended, trough and intra-oral device, to obtain balance of the occlusal forces, to obtain higher occlusal stability during violin playing, which will reflect in a higher articular stability and better balance of the MBPs in the position that the musicians stand for large periods of time [7-10].

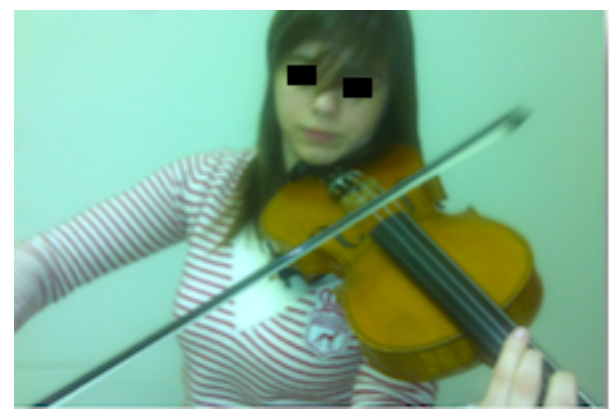

Figure 1. Violin player.

Correspondence to: Dr. Agostinho Martins dos Santos, Faculty of dental medicine university of oporto, Portugal, E-mail: a_sotnas@hotmail.com

Key words: TMJ, TMD, occlusal stability, violin, intra-oral device

Received: March 13, 2016; Accepted: April 22, 2016; Published: April 25, 2016 
The device should be comfortable for continual use, because of the long hours that the violinists spend on practice and, should also, be resistant to withstand the occlusal load that is believed to be generated while stabilizing the violin in the adequate position.

This study saw the idealization and confection of an intra-oral device, with the objective of giving a balance of occlusal forces, by promoting contacts in all of the teeth. This device has an ergonomic design and was made in a way that would promote same intensity contacts in all of the teeth, so that the occlusal force is equally distributed throughout the arches.

\section{Materials and methods}

For the confection of the device Erkoloc-pro plates (Erkodent, Germany), with $3 \mathrm{~mm}$ thick, were utilized $-2 \mathrm{~mm}$ polyethylene terephthalate glycol-modified (PETG) and $1 \mathrm{~mm}$ polyurethane, both of which termomoldable to better individualize the device [11-14]- and autopolymerized poly(methyl methacrylate) (PMMA) as well.

PMMA was chosen to be in contact with the maxillary teeth, for it is biocompatible, insoluble, resistant to occlusal forces and has a chemical bond with PETG $[15,16]$. For the inferior portion of the device, which is in contact with the mandibular teeth, the material should be soft, without jeopardizing the resistance, making the device ergonomic for the user.

The cast acquisition of the dental arches took place in the Occlusion, TMJ and Orofacial Pain department at the Faculty of Dental Medicine of the Oporto University as well as the confection of the intra-oral device.

To test the device, a population of 14 violinists with ages between 18 and 30 years, with a mean of 21.4 years, 12 female and 2 male, was observed.

\section{Inclusion criteria}

Professional violinist or of a superior college of music with, at least, 5 years of activity.

Those that practice daily and for long periods of time.

With age 18 or higher.

With full denture, or the absence of the maximum of two teeth in different hemi arches Ethical Approval: These protocol was approved by the Faculty of Dental Medicine of the Oporto University (FMDUP) ethics committee. The Present study was made after free informed consent of each participant. The main investigator made himself available to clarify any doubt referring the goal of the study and assuring the confidentiality of data and anonymity of each person. Any participant could willing abandon the study at any time.

\section{Method}

- Teeth casts acquisition;

- Register of the maxillary position with the Arcus face bow (KaVo, Germany);

- Intermaxillary relation register, in the most usual playing position, with Occlufast (Zhermack, Italy);

- Teeth cast ensemble in Protar 7 semi-adjustable articulator (KaVo, Germany) with face bow;

- Confection of the Intra-oral device with Erkoloc-pro $3 \mathrm{~mm}$ plates in the Erkoform-3D (Erkodent, Germany) with the use of Occluform-3 (Erkodent, Germany).

- Balancing of the intra-oral device in the semi-adjustable articulator Protar 7 (KaVo, Alemanha) with articulating paper, double faced, dental arch shape $80 \mu \mathrm{m}$ thick (Hanel, U.S.A.) and a computerized device for tridimensional analysis of occlusal contacts T-Scan III (TeKscan, U.S.A.)

- Balancing of the intra-oral device in mouth with the computerized device for tridimensional analysis of occlusal contacts T-Scan III (TeKscan, U.S.A.)

\section{Methods description}

Impression acquisition, of both violinist's arches, with standard perforated metal trays (ASA Dental, Italy) and Orthoprint Fast Set alginate, (Zhermack, Italy) as impression material (Figure 2).

Maxillary arch position register with Arcus face bow (KaVo. Germany) using Godiva Compo (Kemdent, UK) in the bite fork. The violinists were the asked to play, for a while, until they managed to reach their usual playing position while practicing. It was in this mandibular position that we registered the intermaxillary relation in the musical execution of the violinist with Occlufast (Zhermack, Italy) (Figure 3).

The impressions were then set to type III cast to initiate the confection of the device in the Erkoform-3D (Erkodent, Germany) with Occluform-3 (Erkodent, Germany) coupled. The inferior arch cast was placed in the Erkoform-3D (Erkodent, Germany) and the superior cast was placed, according to the intermaxillary register, in the Occluform-3D (Erkodent, Germany). The Occluform pin was set as to remove the intermaxillary register $\mathrm{mm}$ thickness from between

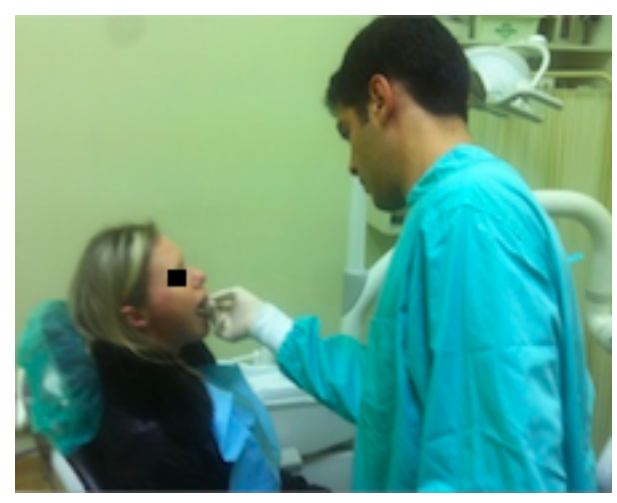

Figure 2. Teeth arch impression.

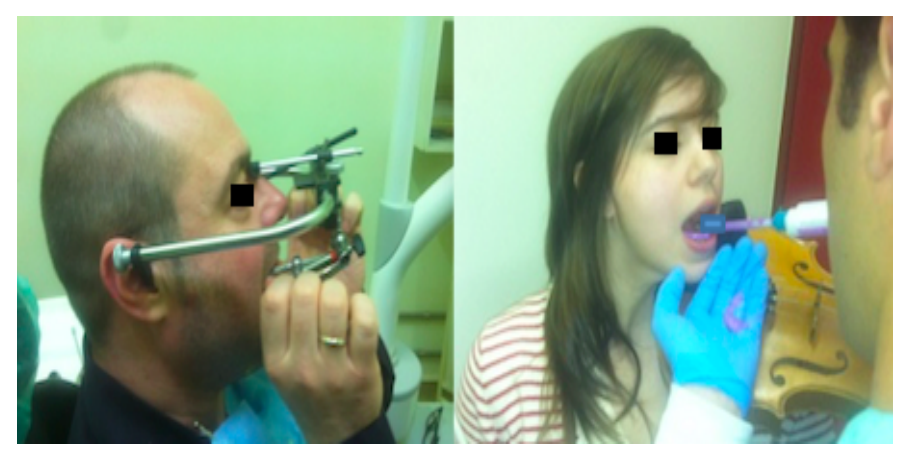

Figure 3. A. Register for Protar 7 with Arcus face bow of the maxillary position (KaVo, Germany). B. Intermaxillary register with Occlufast (Zhermack, Italy). 
the models - measured with Iwanson Decimal Caliper for wax (ASA Dental, Italy).

After thermo molding the Erkoloc-Pro (Erkodent, Germany) $3 \mathrm{~mm}$ thick and $120 \mathrm{~mm}$ diameter plate (Erkodent, Germany) to the inferior cast, we used the Occluform-3 to place the superior cast beneath it to create minimal indentation (less than $1 \mathrm{~mm}$ ) in the plate corresponding to the superior teeth (Figure 4).

The superior cast was ensemble in the semi-adjustable articulator Protar 7 (KaVo, Germany) with plaster of Paris using the Arcus face bow (KaVo, Germany), and the inferior cast was ensemble with the intermaxillary register according to the superior cast. To avoid the vertical expansion of the Paris type plaster we used plastic cable ties around the articulator to maintain the cast ensemble reliable (Figure 5).

Having the casts ensembled in articulator, the thermo molded plate was adjusted with articulating paper, double faced, dental arch shape $80 \mu \mathrm{m}$ thick (Hanel, U.S.A.) by removing divots and by adding PMMA Orthocryl (Dentaurum, Germany) until we reach contacts with all of the superior cast teeth.

The PMMA was set in a pressure cooker with 2 bar pressure and water at $50^{\circ} \mathrm{C}$ for 15 minutes. After set, occlusal balancing was reached by using carbide acrylic burs and hand piece to eliminate first contacts until achieving point mark contacts on the molars, pre-molars and canines and line mark contacts on the incisors.

Finally the occlusal contacts of the device were adjusted in mouth with T-Scan III (TeKscan, U.S.A.) (Figure 6).

\section{Results}

All violinists reported discomfort and muscle aching, both during

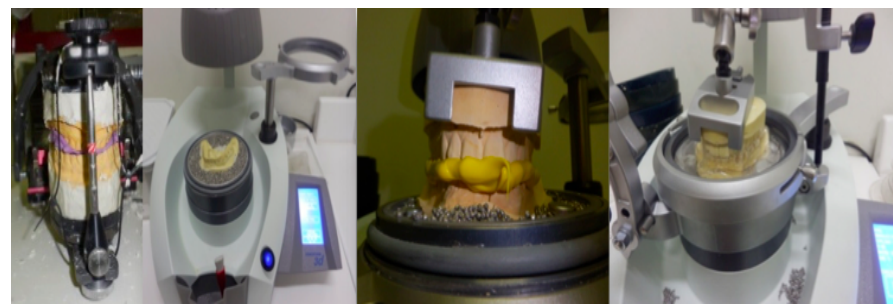

Figure 4. A. Teeth casts ensemble in Protar 7 (KaVo, Germany); B. Erkoform-3D (Erkodent, Germany) and inferior cast; C. Models assembly according to inter-maxillary register; $\mathbf{D}$ Occluform-3 (Erkodent, Germany) and teeth casts.

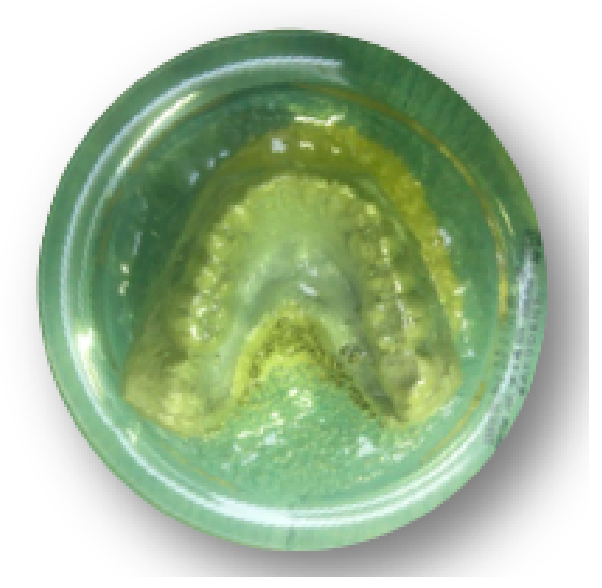

Figure 5. Erkodent termomolded plate (Erkodent, Germany).

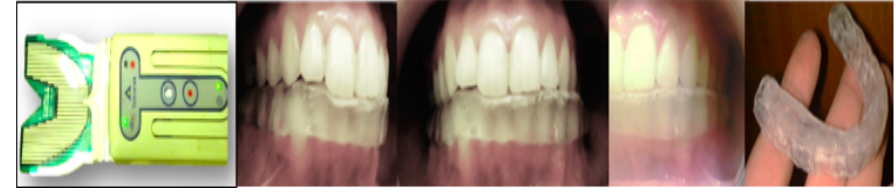

Figure 6. A. T-Scan III (TeKsan, U.S.A.) and leaf; B. Intra-oral device after intraoral adjustment with hand-piece and burs; $\mathbf{C}$. Intraoral device before finishing and polishing.

and after violin practice and playing. All violinists who participated reported that they felt the need of something to alleviate the muscle contraction and pain

As a result we obtained a device that provides occlusal stability was achieved by providing same intensity contacts in all of the teeth - verified with T-Scan. All violinists reported that it managed to avoid teeth grinding and also alleviate muscle tension during violin playing.

Violinists can wear this device during violin playing while it promotes a better distribution of occlusal forces by providing contacts in the teeth on the opposing side of the violin and it does not affect violin playing as reported by the violinist when asked a month after usage.

All violinist reported that, with the device they no longer grinded their teeth, felt the mandible more stable and didn't feel so much contraction, aching or pain in orofacial muscles. They felt all the teeth contacting. When, eventually, they forgot to use the device, they missed it.

\section{Discussion}

With the lateralization of the mandible, violinists concentrate the bite force on a few teeth in the side that rests the violin, having sometime contra-lateral contacts which will lead to long periods of time with articular instability [1].

The majority of violinists in superior schools report, at least, 4 hours of daily practice.

Many of them present muscle hypertonia while at rest, mainly in masseter, trapeze and sternocleidomastoid muscle on the left side [6].

In this study, habitual violin playing position's dental occlusal relation was observed and, also, how it could benefit from occlusal balance as well as provide orofacial muscle tension relief that these musicians feel after long hours of violin playing.

By wearing an intra-oral device, it was intended to achieve occlusal stability while playing and higher balance of muscular forces which can correspond to a muscle relaxation in position that the musicians take.

The device has an ergonomic design and it was intended, that with its usage, the violinist could execute his performance, with his postural and masticatory muscle forces equally distributed, giving stability to the TMJs. The effect of intra-oral occlusal devices has not been entirely clarified and there is no study, up to this moment, that can pinpoint the reason of the relief which they provide. There are several theories that try to explain this mechanism, however, none of them, by themselves, were capable of providing a satisfactory explanation [7-9].

The device was individualized for the most usual playing position, of each violinist, which can be considered parafunctional. Although they oscillate while performing there is, in fact, a position that they hold for most of the time and this is made evident by a characteristic lesion known as fiddler's neck where the stabilization of the violin in a certain position during long periods of time will produce a lesion in the neck [17]. 
The device was idealized for usage only while playing the violin and must be removed while not playing

As mentioned earlier all violinists reported that, when using the device while playing the violin, they no longer grinded their teeth, felt the mandible more stable and didn't feel so much strength or contraction in orofacial muscles. They felt all the teeth contacting better stabilizing the mandible and, consequently, the TMJ.

\section{Conclusion}

Bearing in mind the contingencies of this work, we concluded that the usage of an intra-oral device is needed during violin playing and might prove to be effective in reducing muscle hypertonia and preventing temporomandibular disorders, due to the improvements in occlusal stability given with same intensity contacts throughout all the teeth as well as a relief in symptoms of orofacial muscle pain.

The mechanism of how an intra-oral device actually works was never fully explained although several theories try to explain it. This device was individualized to a certain position that violinists tend to maintain during most of the playing time supported by a lesion called the fiddler's neck, it was also intended to be used during violin playing only.

All violinist reported that the device provided more comfort and more stability of the mandible, as they felt no teeth grinding and less contraction in orofacial muscles.

To clarify this concepts there is a need for more studies.

\section{Ethic responsibilities}

People and animal protection. The authors declare having obtained previous approval for the protocols from their work center in accordance with the world medical association declaration of Helsinki for medical research involving human subjects

Data confidentiality. The authors declare having followed the protocols of their work center when it comes to publishing data.

Right to privacy and informed written consent. The authors declare having received a written consent. The corresponding author is in possession of these documents.

\section{Conflict of interest}

This research was carried without funding. No conflict of interests declared.

\section{References}

1. al-Ani MZ, Gray RJ, avies SJ, Sloan P (2003) A study of the relationship between lateral guidance and temporomandibular joint internal derangement. Eur J Prosthodont Restor Dent 11: 65-70. [Crossref]

2. McKay GS, Yemm R, Cadden SW (1992) The structure and function of the temporomandibular joint. Br Dent J 173: 127-132. [Crossref]

3. Dawson PE (1979) Centric relation. Its effect on occluso-muscle harmony. Dent Clin North Am 23: 169-180. [Crossref]

4. Alarcon JA, Martin C, Palma JC, Menéndez-Núñez M (2009) Activity of jaw muscles in unilateral cross-bite without mandibular shift. Arch Oral Biol 54: 108-114. [Crossref]

5. Collaer P, Linden AV (1968) Historical atlas of music; a comprehensive study of the world's music, past and present. Cleveland, World Pub. Co.

6. Lee SH, Carey S, Dubey R, Matz R (2012) Intervention program in college instrumenta musicians, with kinematics analysis of cello and flute playing: a combined program of yogic breathing and muscle strengthening-flexibility exercises. Med Probl Perform Art 27: 85-94. [Crossref]

7. Alencar F Jr, Becker A (2009) Evaluation of different occlusal splints and counselling in the management of myofascial pain dysfunction. J Oral Rehabil 36: 79-85. [Crossref]

8. Fricton J (2006) Current evidence providing clarity in management of temporomandibular disorders: summary of a systematic review of randomized clinical trials for intra-oral appliances and occlusal therapies. J Evid Based Dent Pract 6: 4852. [Crossref]

9. Wang XR, Zhang Y, Xing N, Xu YF, Wang MQ (2013) Stable tooth contacts in intercuspal occlusion makes for utilities of the jaw elevators during maximal voluntary clenching. J Oral Rehabil 40: 319-328. [Crossref]

10. Wieczorek A, Loster J, Loster BW (2013) Relationship between occlusal force distribution and the activity of masseter and anterior temporalis muscles in asymptomatic young adults. Biomed Res Int 35-40.

11. Vallittu PK, Narva K (1997) Impact strength of a modified continuous glass fiberpoly(methyl methacrylate). Int J Prosthodont. 10: 142-148. [Crossref]

12. Stoyko F (2002) Handbook of Thermoplastic Polyesters. Wiley-VCH, Weinheim: 1223.

13. Woods G (1990) The ICI Polyurethanes Book. John Wiley \& Sons, Inc New York

14. Corina M (2014) Advantages of a Polyethylene Terephthalate Glycol-modified Coated with a Thermoplastic Polyurethane as an Occlusal Appliance Material. REV. CHIM. (Bucharest).

15. Jagger RG, Milward PJ, Jagger DC, Vowles RW (2003) Accuracy of adaptation of thermoformed poly(methyl methacrylate) J Oral Rehabil. 30: 364-368. [Crossref]

16. Steele JG, Wassell RW, Walls AW (1992) A comparative study of the fit and retention of interocclusal splints constructed from heat-cured and autopolymerized polymethylmethacrylate. J Prosthet Dent 67: 328-330. [Crossref]

17. Goldstein LJ (1968) When violin scars jaw, try custom chin rest. Dent Surv 44: 48-49. [Crossref]

Copyright: (C2016 Santos AM. This is an open-access article distributed under the terms of the Creative Commons Attribution License, which permits unrestricted use, distribution, and reproduction in any medium, provided the original author and source are credited. 